This document is confidential and is proprietary to the American Chemical Society and its authors. Do not copy or disclose without written permission. If you have received this item in error, notify the sender and delete all copies.

\title{
Ultrasensitive label-free nanosensing and high-speed tracking of single proteins
}

\begin{tabular}{|r|l|}
\hline Journal: & Nano Letters \\
\hline Manuscript ID & nl-2016-05040z.R2 \\
\hline Manuscript Type: & Communication \\
\hline Complete List of Authors: & $\begin{array}{l}\text { Liebel, Matz; ICFO - The Institute of Photonic Sciences, The Barcelona } \\
\text { Institute of Science and Technology } \\
\text { Hugall, James; ICFO - The Institute of Photonic Sciences, The Barcelona } \\
\text { Institute of Science and Technology } \\
\text { van Hulst, Niek; ICFO - The Institute of Photonic Sciences, The Barcelona } \\
\text { Institute of Science and Technology, }\end{array}$ \\
\hline
\end{tabular}

SCHOLARONE

Manuscripts 


\title{
Ultrasensitive label-free nanosensing and high-speed tracking of single proteins
}

\author{
Matz Liebel ${ }^{1}$, James T. Hugall ${ }^{1}$, and Niek F. van Hulst ${ }^{1,2, *}$ \\ ${ }^{1}$ ICFO-Institut de Ciencies Fotoniques, The Barcelona Institute of Science and Technology, \\ 08860 Castelldefels, Barcelona, Spain \\ ${ }^{2}$ ICREA-Institució Catalana de Recerca i Estudis Avançats, 08010 Barcelona, Spain
}

\begin{abstract}
Label-free detection, analysis and rapid tracking of nanoparticles is crucial for future ultrasensitive sensing applications, ranging from understanding of biological interactions to the study of size dependent classical-quantum transitions. Yet optical techniques to distinguish nanoparticles directly amongst their background remain challenging. Here we present amplified interferometric scattering microscopy (a-iSCAT) as a new all-optical method capable of detecting individual nanoparticles as small as $\mathbf{1 5} \mathrm{kDa}$ proteins which is equivalent to half a GFP. By balancing scattering and reflection amplitudes the interference contrast of the nanoparticle signal is amplified one to two orders of magnitude. Beyond high sensitivity, a-iSCAT allows high-speed image acquisition exceeding several hundreds of frames-per-second. We showcase the performance of our approach by detecting single Streptavidin binding events and by tracking single Ferritin proteins at $\mathbf{4 0 0}$ frames-per-second with $\mathbf{1 2} \mathrm{nm}$ localization precision over seconds. Moreover, due to its extremely simple experimental realisation, this advancement finally enables a cheap and routine implementation of label-free all-optical single nanoparticle detection platforms with sensitivity operating at the single protein level.
\end{abstract}

KEYWORDS: interferometric scattering; balanced interference; label-free; single particle tracking; single-molecule detection; superresolution; iSCAT

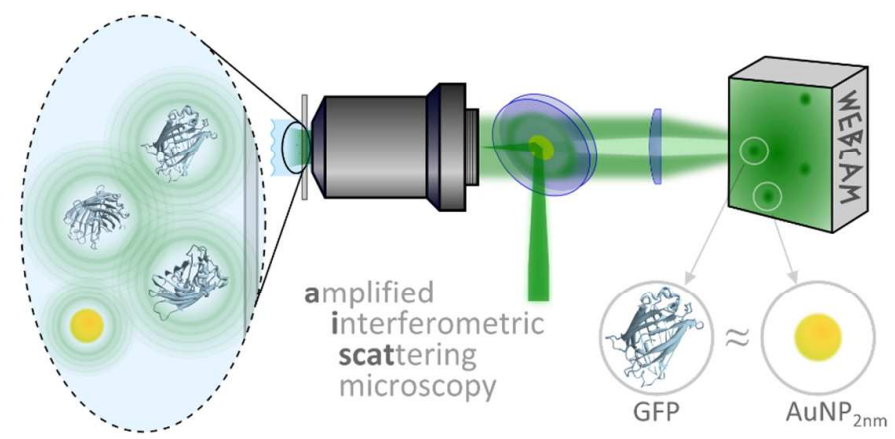

TOC Figure: Amplified interferometric scattering microscopy (a-iSCAT) enables label-free detection of individual nanoparticles as small as $15 \mathrm{kDa}$ proteins or a $2 \mathrm{~nm}$ diameter gold sphere. 
Over the past two decades single-particle localization microscopy has contributed enormously to our understanding of biological processes (1-6). All of these methods rely on the indirect detection of the species of interest by labelling it with either a fluorophore or large scattering particle. Fluorescence has the advantage of being an essentially background-free process, yet provides only a limited number of photons, limiting localisation precision, and suffers from fluorophore photobleaching and blinking events $(3,7,8)$. Scattering based techniques, such as darkfield microscopy, are not limited by these constraints, labels however commonly exceed several tens of nanometres to be distinguishable from background scattering $(2,5,9)$. Both approaches, however, require complicated labelling strategies and controls to ensure that the labels do not perturb the biological processes under study (10-12). Recently, two groups have demonstrated that interferometric scattering microscopy (iSCAT) $(13,14)$ is able to detect and even track single proteins in a label free manner $(15,16)$. These proof-of-concept experiments confirmed the potential of alloptical detection methods but required microscopes incorporating confocal beam scanning and PID controlled laser systems, while the shallow protein contrast $\left(10^{-4}-10^{-3}\right)$ demanded expensive high dynamic range cameras with large full well capacities. This experimental complexity in combination with the low imaging frame rates achieved, has hampered their applicability to biological questions.

Here we introduce amplified interferometric scattering microscopy (a-iSCAT) that surpasses the previously reported protein detection bandwidth by more than an order of magnitude by attenuating unwanted reference signals, pushing sensitivity and imaging speed to relevant biological conditions, while simultaneously relying on a drastically simplified optical setup. a-iSCAT allows direct observation of individual bio-particles: single proteins, extracellular vesicles, antigens, etc., with a sensitivity of $15 \mathrm{kDa}$, which is a third of a single GFP protein. Beyond protein detection in its current form a-iSCAT is capable of detecting clusters of a few hundreds of atoms thus enabling alloptical studies on the transition between classical and quantum in metallic nanostructures (17).

\section{Results}

In brief, the sample, commonly contained in water on top of a glass coverslip, is wide-field illuminated by focusing the output of a laser diode onto the back-focal-plane of an oil immersion objective via a partially reflective metallic mask coated onto the centre of a glass window (Figure 1a, see Methods for fabrication details). Fractions of the illumination light are either reflected at the glass-water interface or scattered by particles within the illuminated area. Both the scattered and the reflected electric field components are collected by the objective and exit the back-focal-plane where they interact with the partially mirrored mask mounted in its proximity. The scattering from the sample is predominantly concentrated towards the critical angle and thus emerges from the back-focal-plane as a ring towards the edge of the objective, and is transmitted by the uncoated part of the mask (Figure 1b) (18). The electric field reflected from the glass-water interface, however, propagates collinear with the illumination beam and its amplitude is attenuated approximately 50 times by the partially reflective mask in the centre of the window, rather than fully blocked as in dark-field microscopy (9). Importantly, contrary to darkfield microscopy both contributions remain phase-locked and maintain their propagation properties thus leading to interference when imaged onto a CMOS camera where they give rise to the a-iSCAT signal:

$$
I_{a-i S C A T}=I_{0}\left(\frac{r^{2}}{\alpha^{2}}+s^{2}+\frac{2 r s}{\alpha} \cos (\theta)\right)
$$


a

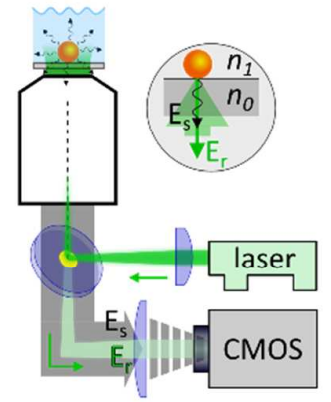

b

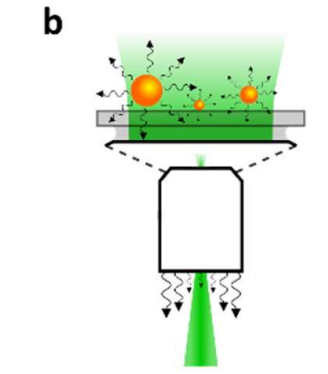

with $I_{0}$ being the illumination intensity, $r$ and $s$ the normalised reflection and scattering amplitudes, $\theta$ the phase difference between $r$ and $s$ and $\alpha$ the attenuation amplitude defined as the reciprocal transmission amplitude. For very small particles one can neglect the pure scattering term with $\frac{r^{2}}{\alpha^{2}} \gg s^{2}$. The a-iSCAT signal is given by $\frac{2 r s}{\alpha} \cos (\theta)$ and all background contributions are contained in the reflection term $\frac{r^{2}}{\alpha^{2}}$ and we are hence able to define the signal-to-background ratio (SBR) as:

$$
\operatorname{SBR}=\alpha \frac{2 \mathrm{~s}}{\mathrm{r}} \cos (\theta),
$$

and the resulting a-iSCAT contrast as the SBR minus one which exhibits the same, linear, volume dependency as iSCAT but with an $\alpha$-fold improvement in contrast (15).

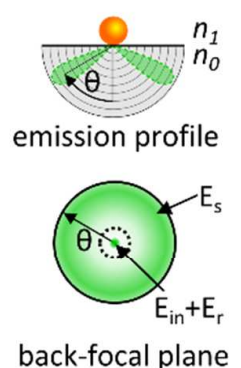

Figure 1, Concept and experimental implementation of amplified interferometric scattering microscopy (a-iSCAT). (a) The sample is widefield illuminated and light scattered from it is collected together with a phase-locked reflection from the glass-water interface. Only a small fraction is transmitted by a mask mounted in the proximity of the back-focal-plane, whereas the scattering passes mostly unattenuated. Both contributions are imaged onto a camera where interference takes place. (b) Widefield illumination is achieved by focusing the laser beam into the centre of the backfocal plane of the microscope objective. Scattering from particles near the glass-water interface is predominantly radiated into the high refractive index material and is concentrated towards the critical angle. The combination of illumination and scattering properties leads to a separation of the respective electric fields in the back-focal plane of the microscope objective. The mask projection onto the back-focal plane is indicated as a dotted circle.

We quantify the performance of a-iSCAT and its superior contrast by recording non-specific singleprotein binding events to a bare cover-glass for 53 to $440 \mathrm{kDa}$ mass proteins. Representative images in Figure 2a show single proteins with contrast at the percent level (-1\% to $-6 \%)$. See Methods and Supplementary Information 1 for a-iSCAT image generation and Supplementary Videos 1 and 2 for non-specific IgG binding events and a control. Contrast histograms obtained from binding events recorded over 3 min reflect the predicted linear protein volume, or mass, dependency of the a-iSCAT signal, typically $-1 \%$ per $100 \mathrm{kDa}$ (Figure $2 \mathrm{~b}, \mathrm{c}$ ).

This experimentally obtained relationship allows us to estimate the detection limit of a-iSCAT from a control sample containing buffer only. Here, fluctuations are due to intrinsic experimental limitations and residual landing events are a result of possible contaminations. By monitoring the residual noise under these conditions we are able to determine the smallest detectable contrast as a function of frame averaging. The detection limit scales shot-noise like up to approximately 100 frame averages, equivalent to 4 frames-per-second (FPS) at our current acquisition rate, when it reaches a sensitivity corresponding to a $15 \mathrm{kDa}$ protein (Figure 2d). Averaging for longer periods does not significantly improve the signal, most likely due to the presence of low frequency vibrations or sample drift, thus resulting in a detection limit of $15 \mathrm{kDa}$, the equivalent of 20 basepairs, at 4 FPS. 
a

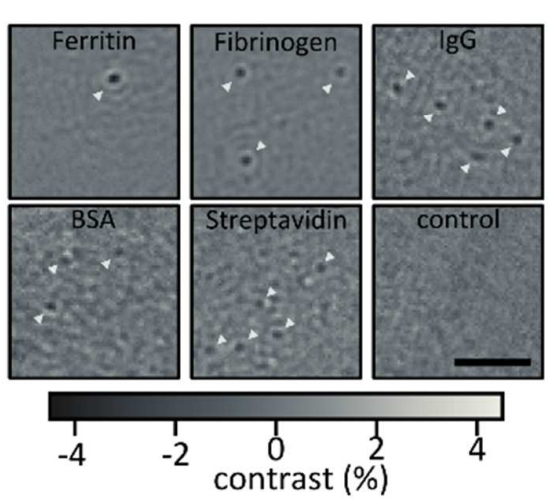

b

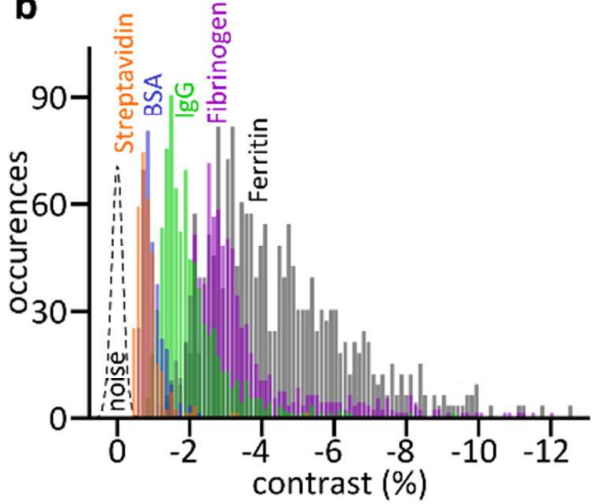

C

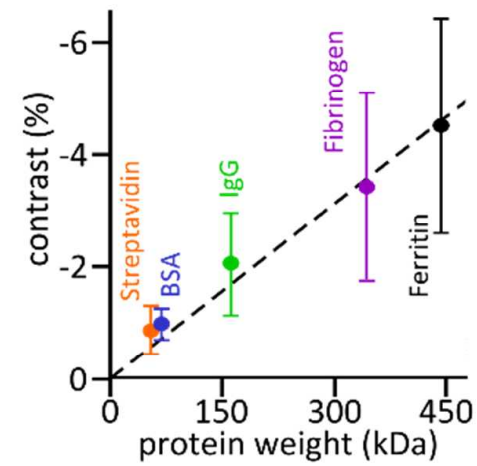

d

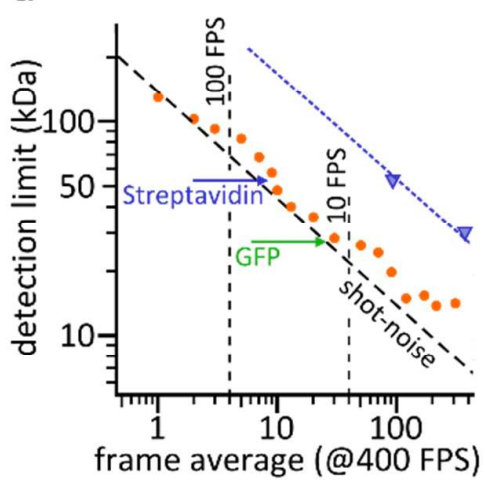

Figure 2, Sensitivity and detection limit of a-iSCAT. (a) a-iSCAT signals obtained for non-specific binding of a variety of single proteins to a cover-glass in comparison to a control with buffer only. To enhance clarity, we generated the images by dividing two consecutive frame averages of 400 frames each but remark that lower frame averages are possible (see Supplementary Information 1). Scale bar (identical for all) $2 \mu \mathrm{m}$. The contrast of IgG, BSA, Streptavidin and control is scaled by a factor of three for clarity. (b) Contrast histograms obtained by counting non-specific single-protein binding events for a total of three minutes per protein, the Ferritin occurrences are magnified by a factor of three. A contrast distribution function for neat buffer is shown for comparison (dashed line). (c) Mean contrast of the five proteins as a function of protein weight alongside a linear fit (dashed line), error bars at $1 \sigma$ confidence interval. (d) Detection limit as a function of frame averaging (orange dots) in comparison to shot-noise-limited behaviour (dashed line). The acquisition rate for all experiments was 400 FPS. The dotted line and triangles show a comparison to the detection limit extracted from work performed with conventional iSCAT (15).

Our detection modality is crucially dependent on being able to resolve small changes between successive frames on top of the background. To reveal non-specific protein binding as described previously it is sufficient to divide frame averages obtained before and after the event of interest. Tracking dynamic events over longer time-scales, such as protein membrane diffusion or the processive motion of molecular motors, requires a waiting time between the two averages to allow for the protein to move from its previous location. Without this waiting time the signal disappears in the division. Depending on the velocity of the process of interest long-term stability of the a-iSCAT, ideally over seconds, becomes a crucial parameter. 
a
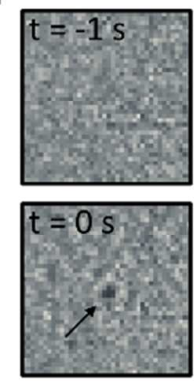

$t=3 s$

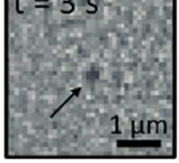

d

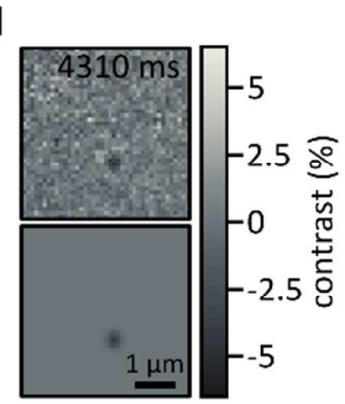

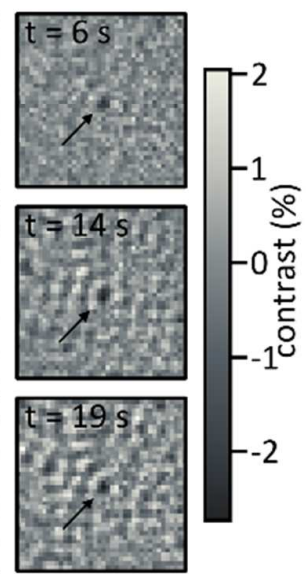

b
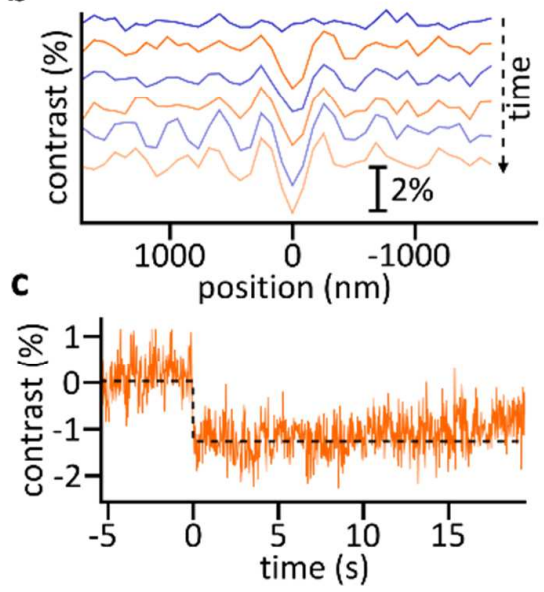

e
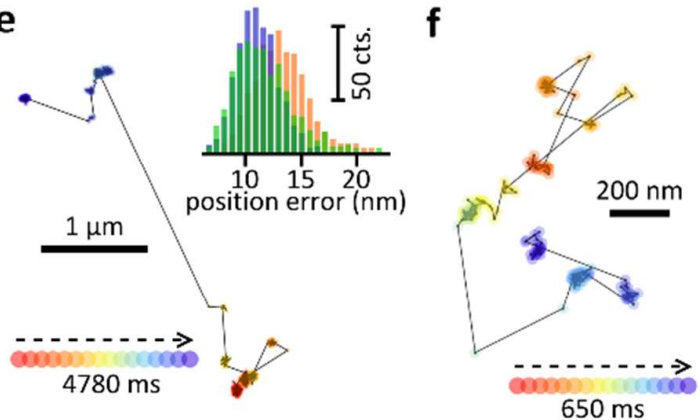

Figure 3, High-speed tracking and long term stability of a-iSCAT. (a) a-iSCAT images of a single IgG protein binding non-specifically to a cover-glass surface at different time delays with respect to the landing event. The images are obtained by averaging 20 frames resulting in an effective imaging rate of 20 FPS and dividing them by an average taken before the landing event at $t=-0.05 \mathrm{~s}$. (b) Crosssections of the IgG signal shown in (a) emphasising the deteriorating signal-to-noise ratio with increasing frame average to background time delays. The respective cross sections have been offset for clarity. (c) Temporal evolution of an a-iSCAT signal obtained for a single IgG protein binding nonspecifically to the coverglass recorded at an effective imaging rate of 40 FPS (10 frame averages) computed with respect to a frame acquired at $t=-6 \mathrm{~s}$. (d) a-iSCAT image acquired within $2.5 \mathrm{~ms}$ (corresponding to 400 FPS) of a diffusing Ferritin protein (top) and Gaussian fit (bottom) computed with respect to a mean background acquired over 10 frames prior to the high speed tracking. The time delay with respect to the background is shown in the inset. (e) Trajectory of the same, single Ferritin protein, obtained analogous to (d), from Supplementary Video 3 by Gaussian fitting of each of the 2000 frames acquired. The total tracking time with respect to the background is indicated by the colour scale. Inset: Histogram of the lateral localisation precisions obtained for the Gaussian fits for the first (orange), second (green) and third (blue) part of the trajectory, with mean precision $\left(\sqrt{\sigma_{x}^{2}+\sigma_{y}^{2}}\right)$ of $12 \mathrm{~nm}$ demonstrate essentially shot-noise limited localisation over the full observation period. (f) As (e) but for a Ferritin protein exhibiting a different diffusion behaviour on the glass surface.

Due to its extremely minimalistic implementation (Figure 1a) a-iSCAT fulfils this stability requirement and is able to detect and follow single IgG binding events over an extended period of time without reacquiring a background frame (Figure $3 a$ ). 
The image remains mostly unchanged and only for frames acquired more than $6 \mathrm{~s}$ after the original background measurement does sample drift-induced noise deteriorate the image quality as well as the signal point-spread-function (Figure 3a, b). Throughout the observation window the achievable localisation precision decreases from initially $10.4 \mathrm{~nm}$ to $17.8 \mathrm{~nm}$ after $19 \mathrm{~s}$. Notably, it is possible to resolve and monitor the immobilisation of single IgG proteins on a cover-glass at 40 FPS over the course of $25 \mathrm{~s}$ with only minor changes in a-iSCAT contrast (Figure 3c).

Beyond the detection of static events with $15 \mathrm{kDa}$ sensitivity limit, a-iSCAT microscopy is well suited for monitoring dynamic biological processes. We therefore conclude with a proof-of-concept experiment mimicking rapid diffusion of a membrane protein. Contrary to the complete immobilisation of IgG, Ferritin only transiently binds to the cover-glass surface and we are therefore able to follow its motion on the substrate. Here we track a single Ferritin at 400 FPS over $5 \mathrm{~s}$ without re-acquiring a new background with essentially shot-noise-limited sensitivity (Figure $3 \mathrm{~d}$ and Supplementary Video 3), and with the observation period only being limited by the unbinding of the protein. Analogous to fluorescence localization microscopy it is possible to determine the precise position of the protein by Gaussian fitting (Figure 3d) (19).

The reconstructed protein trajectory reveals areas of almost fully confined motion reminiscent of trapping events that are interconnected by very fast diffusion, most likely due to complete unbinding of the protein from the glass surface (Figure 3e) (20). We achieve a mean lateral localization precision of $12 \mathrm{~nm}$ (21) (Figure 3e, inset) and remark that the mean protein a-iSCAT contrast of $-4.04 \%$ is in very good agreement with the value expected for the mass of Ferritin (Figure 2c). For some of the Ferritin proteins we observe Brownian motion like diffusion with comparable localization precision and contrast values an example of which is shown in Figure $3 f$ and Supplementary Video 4.

\section{Discussion}

By combining the concept of small signal amplification by phase-locked interference detection with a carefully balanced reference field we are able to dramatically increase the sensitivity of current detection schemes, thereby drastically reducing cost and experimental complexity. We have demonstrated that a-iSCAT microscopy is well suited for studying dynamic events of medium sized proteins at video-rate and faster. This advancement will finally enable the direct label free study of protein-protein interactions at the single particle level, thus removing any uncertainty regarding whether the presence and attachment of fluorescent or scattering labels interferes with normal biological functions. Additionally, the absolute mass sensitivity allows the direct monitoring of assembly processes of, for example, protein complexes such as actin filaments or DNA double helices. To address the full spectrum of biomolecules, especially in the increasingly relevant small protein level (22), we are actively working on extending the a-iSCAT sensitivity down to the sub-kDa level as well as creating an a-iSCAT implementation for commercially available microscopes by relying on recently developed low-profile extensions (23). Due to its drastically reduced complexity, a-iSCAT has the potential to democratize the detection and analysis of nanoparticles by making cost efficient all-optical label-free sensors widely accessible. Even though our proof of concept experiments focused on proteins due to their precisely defined molecular weight the results obtained can be directly translated to biomarkers or metallic nanoparticles. For example, a-iSCAT is approximately five orders of magnitude more sensitive than the commercially available and widely used plasmonic detection platform relying on enzyme-linked immunosorbent assays (ELISA) thus enabling single biomarker detection and analysis for improved point-of-care diagnostics. We envision a considerable impact of our technique in the emerging field of extracellular vesicles such as exosomes as highly sensitive methods for quantitative size determination are highly sought after 
but still lacking (24-28). Moreover a-iSCAT not only enables a reliable size determination of extracellular vesicles by combining its intrinsic molecular weight dependence with the possibility to determine the particle radius by nanoparticle tracking analysis but, additionally, will allow for the study of such vesicles on a single particle level (29). Our detection limit is a single $15 \mathrm{kDa}$ protein exhibiting a signal equivalent to that of a $1.6 \mathrm{~nm}$ diameter gold sphere $(16,30)$. With such sensitivity the a-iSCAT approach is a promising alternative to directly access the regime where quantum size effects govern the electromagnetic response of plasmonic structures by taking advantage of Fourier transform spectroscopy to determine the size dependent scattering spectrum of the observed particle $(17,31,32)$.

\section{Methods}

Fabrication of the a-iSCAT mask. One-inch vinyl stickers with elliptical holes of $1.41 \mathrm{~mm} \times 2 \mathrm{~mm}$ (aspect ratio $1: 1$ at $45^{\circ}$ illumination angle) cut into their centre are attached to $5 \mathrm{~mm}$ thick antireflection coated (A-coated) BK7 windows (Thorlabs WG11050-A) to act as an evaporation mask. Metal ( $5 \mathrm{~nm}$ of Titanium at a rate of $0.1 \mathrm{~nm}$ per second followed by $160 \mathrm{~nm}$ of Gold at a rate of 0.2 $\mathrm{nm}$ per second) is then deposited onto the windows using a Lesker LAB18 evaporator. The thickness corresponds to an approximate transmission of $3 \times 10^{-4}$ at $520 \mathrm{~nm}$. Upon removal of the vinyl sticker the a-iSCAT mask is directly used in the microscope. The transmission properties for masks fabricated from different metals or at different thicknesses/illumination wavelengths are easily computed from the material's complex dielectric constant, such as found in the refractiveidex.info database.

Optical implementation. The linearly polarised output of a Roithner Lasertechnik NLD521000G 520 $\mathrm{nm}$ laser diode amplitude modulated at $10 \mathrm{MHz}$ is focused onto a $50 \mu \mathrm{m}$ pinhole and the emerging light re-collimated by a $30 \mathrm{~mm}$ focal length lens. A $300 \mathrm{~mm}$ focal length lens focuses the beam onto the back focal plane of a home-built inverted microscope, equipped with an Olympus APON 60XOTIRF objective (NA=1.49, similar results are obtained with a Olympus 100XUPLSAPO; NA=1.4), by reflecting off the a-iSCAT mask described above. The signal emerging from the objective is transmitted through the a-iSCAT mask and focused onto a Point Grey CMOS camera by means of a $400 \mathrm{~mm}$ focal length lens positioned one tube length away from the back focal plane of the microscope objective (Grasshopper3 2.3 MP Mono USB3 Vision: 400 FPS, 256x256 pixel field of view at a resolution of $43 \mathrm{~nm} /$ pixel resulting in an approximate field of view of $11 \times 11 \mu \mathrm{m}$, digital $2 \times 2$ pixel binning is applied in all experiments). The illumination intensity is adjusted to be $33 \mathrm{~kW} / \mathrm{cm}^{2}$ at the image plane. XY-translation of the sample is achieved with a manual translation stage (OptoSigma), Z-translation adjustment of the focus by means of a manual focusing block (PRIOR Scientific, FB201 Focus Block). No active stabilisation is present.

Data processing and analysis. To generate the a-iSCAT images we average over a given number of raw images acquired at 400 FPS to generate a background image followed by an image containing the signal of interest. The latter is then divided by the former in order to obtain the differential contrast a-iSCAT image. Unless stated otherwise, directly adjacent frame averages are divided (e.g. the average of frames 10-19 divided by the average of frames 0-9). Here, we do not report any images obtained by dividing averages with partially overlapping frames to avoid incorrect contrast and noise estimates. All protein landing events presented in Figure 2 are identified by eye. To avoid 
biased selection of a certain contrast level we randomise the data sets of all five proteins prior to analysis. Controls with pure buffer solutions are performed prior to all experiments.

Non-specific single protein binding experiments. Fibrinogen (Human type I from human plasma), Ferritin (From equine spleen, type I), BSA, IgG (From mouse serum) and PBS buffer were purchased from Sigma Aldrich. ATTO647N-labeled Streptavidin was purchased from ATTO-TEC GmbH. Prior to each experiment we clean the cover-glass by sonication in acetone and milliQ (10 min each) followed by drying under a flow of nitrogen and 20 min of UV-Ozone cleaning (Bioforce ProCleanerPlus). Silicone isolators (Grace Bio-Labs) are placed on the freshly cleaned cover-glass, to form sample chambers, and filled with $40 \mu \mathrm{l}$ of aqueous buffer (PBS for BSA, IgG, Ferritin and Fibrinogen) or milliQ water (Streptavidin). To verify that binding events originate from proteins we first monitor the signal level of neat solvent over the course of three minutes after which we add $2 \mu \mathrm{l}$ of approx. $100 \mathrm{pM}$ protein solution, in its respective solvent, to the $40 \mu \mathrm{l}$ of clean buffer/milliQ water and start detecting binding events.

Acknowledgements. We would like to thank Luis Enrique, Johann Osmond, Ion Hancu and the Tinkerers Lab [Fab Lab] for helping us with the fabrication of the a-iSCAT mask, Xavier Menino and the whole mechanical workshop for the construction of the microscope torso and Dr. Jaime OrtegaArroyo for helpful discussions throughout the project. This research was funded by the European Commission (ERC Adv. Grant 670949-LightNet); MINECO Severo Ochoa Programme for Centres of Excellence in R\&D (SEV-2015-0522), FIS2012-35527, FIS2015-69258-P; Catalan AGAUR (2014 SGR01540); CERCA Programme of Generalitat de Catalunya; and Fundació CELLEX (Barcelona). M.L. acknowledges financial support from the Marie-Curie International Fellowship COFUND.

Supporting Information. Details on a-iSCAT image formation. This material is available free of charge via the Internet at http://pubs.acs.org.

\section{References.}

1. Betzig, E. Proposed method for molecular optical imaging. Opt. Lett. 20, 237-9 (1995).

2. Saxton, M. J. \& Jacobson, K. Single-particle tracking: applications to membrane dynamics. Annu. Rev. Biophys. Biomol. Struct. 26, 373-399 (1997).

3. Moerner, W. E. \& Orrit, M. Illuminating Single Molecules in Condensed Matter. Science 283, 1670-1676 (1999).

4. Betzig, E. et al. Imaging intracellular fluorescent proteins at nanometer resolution. Science 313, 1642-1645 (2006).

5. Nan, X., Sims, P. A. \& Xie, X. S. Organelle tracking in a living cell with microsecond time resolution and nanometer spatial precision. ChemPhysChem 9, 707-712 (2008).

6. Xu, K., Zhong, G. \& Zhuang, X. Actin, spectrin, and associated proteins form a periodic cytoskeletal structure in axons. Science 339, 452-6 (2013).

7. Dickson, R. M., Cubitt, a B., Tsien, R. Y. \& Moerner, W. E. On/off blinking and switching behaviour of single molecules of green fluorescent protein. Nature 388, 355-358 (1997).

8. Tinnefeld, P., Herten, D. \& Sauer, M. Photophysical Dynamics of Single Molecules Studied by Spectrally-Resolved Fluorescence Lifetime Imaging Microscopy (SFLIM). J. Phys. Chem. A 105, 7989-8003 (2001). 
9. Weigel, A., Sebesta, A. \& Kukura, P. Dark Field Microspectroscopy with Single Molecule Fluorescence Sensitivity. ACS Photonics 1, 848-856 (2014).

10. Clausen, M. P. \& Lagerholm, B. C. The Probe Rules in Single Particle Tracking. Curr. Protein Pept. Sci. 12, 699-713 (2011).

11. Greenleaf, W. J., Woodside, M. T. \& Block, S. M. High-resolution, single-molecule measurements of biomolecular motion. Annu. Rev. Biophys. Biomol. Struct. 36, 171-190 (2007).

12. Landgraf, D., Okumus, B., Chien, P., Baker, T. a \& Paulsson, J. Segregation of molecules at cell division reveals native protein localization. Nat. Methods 9, 480-482 (2012).

13. Lindfors, K., Kalkbrenner, T., Stoller, P. \& Sandoghdar, V. Detection and spectroscopy of gold nanoparticles using supercontinuum white light confocal microscopy. Phys. Rev. Lett. 93, 037401-1 (2004).

14. Kukura, P. et al. High-speed nanoscopic tracking of the position and orientation of a single virus. Nat. Methods 6, 923-927 (2009).

15. Piliarik, M. \& Sandoghdar, V. Direct optical sensing of single unlabelled proteins and superresolution imaging of their binding sites. Nat. Commun. 5, 4495 (2014).

16. Ortega Arroyo, J. et al. Label-free, all-optical detection, imaging, and tracking of a single protein. Nano Lett. 14, 2065-2070 (2014).

17. Scholl, J. A., Koh, A. L. \& Dionne, J. A. Quantum plasmon resonances of individual metallic nanoparticles. Nature 483, 421-427 (2012).

18. Novotny, L. \& Hecht, B. U. of B. Principles of Nano-Optics. 2, (2012).

19. Crocker, J. C. \& Grier, D. G. Methods of Digital Video Microscopy for Colloidal Studies. J. Colloid Interface Sci. 179, 298-310 (1996).

20. Ritchie, K. et al. Detection of non-Brownian diffusion in the cell membrane in single molecule tracking. Biophys. J. 88, 2266-2277 (2005).

21. Deschout, H. et al. Precisely and accurately localizing single emitters in fluorescence microscopy. Nat. Methods 11, 253-266 (2014).

22. Storz, G., Wolf, Y. I. \& Ramamurthi, K. S. Small proteins can no longer be ignored. Annu. Rev. Biochem. 83, 753-77 (2014).

23. Ishmukhametov, R. R., Russell, A. N., Wheeler, R. J., Nord, A. L. \& Berry, R. M. A Simple lowcost device enables four epi-illumination techniques on standard light microscopes. Sci. Rep. 6, 20729 (2016).

24. Anastasiadou, E. \& Slack, F. J. Malicious exosomes. Science 346, 1459-1460 (2014).

25. Kaiser, J. Malignant messengers. Science 352, 164-166 (2016).

26. Im, H. et al. Label-free detection and molecular profiling of exosomes with a nano-plasmonic sensor. Nat. Biotechnol. 32, 490-5 (2014).

27. van der Pol, E., Coumans, F., Varga, Z., Krumrey, M. \& Nieuwland, R. Innovation in detection of microparticles and exosomes. J. Thromb. Haemost. 11, 36-45 (2013).

28. Hoen, E. N. M. N. t et al. Quantitative and qualitative flow cytometric analysis of nanosized cell-derived membrane vesicles. Nanomed. Nanotech. Biol. Med. 8, 712-720 (2012).

29. Dragovic, R. A. et al. Sizing and phenotyping of cellular vesicles using Nanoparticle Tracking Analysis. Nanomed. Nanotech. Biol. Med. 7, 780-788 (2011).

30. Jacobsen, V., Stoller, P., Brunner, C., Vogel, V. \& Sandoghdar, V. Interferometric optical detection and tracking of very small gold nanoparticles at a water-glass interface. Opt. 
Express 14, 405-414 (2006).

31. Tame, M. S. et al. Quantum plasmonics. Nat. Phys. 9, 329-340 (2013).

32. Piatkowski, L., Gellings, E. \& van Hulst, N. F. Broadband single-molecule excitation spectroscopy. Nat. Commun. 7, 10411 (2016). 


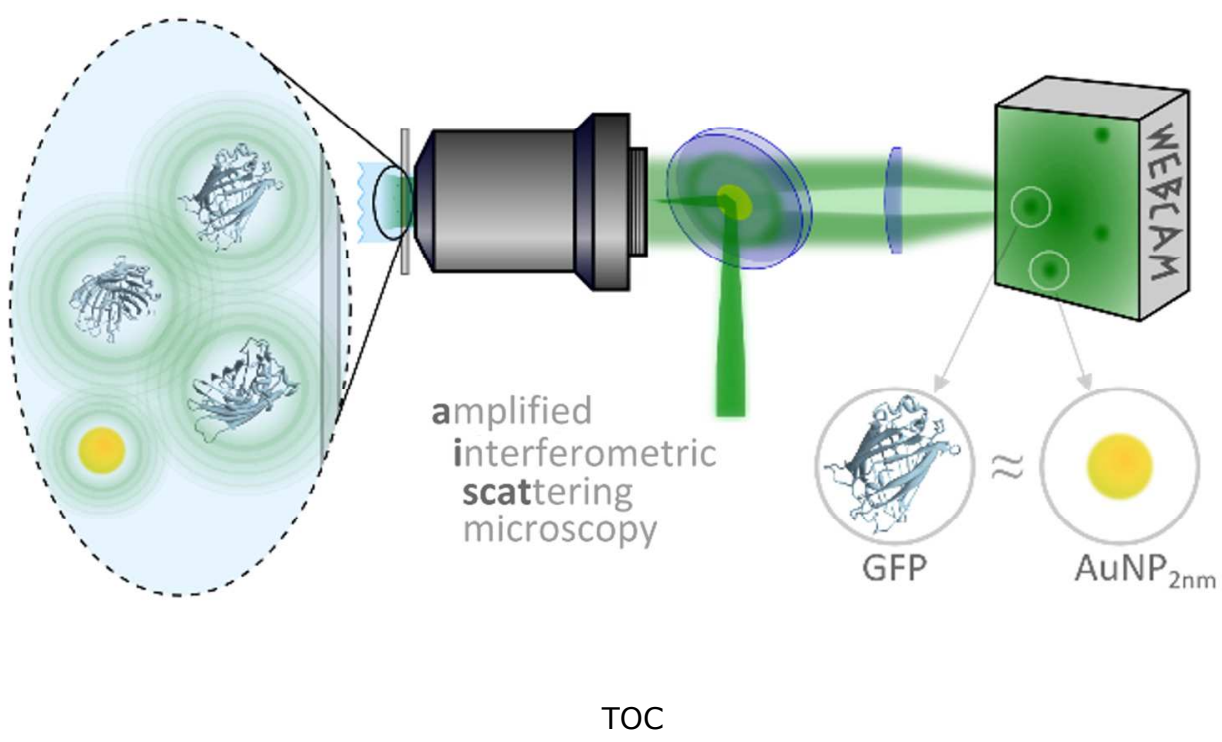

$307 \times 154 \mathrm{~mm}(150 \times 150 \mathrm{DPI})$ 